\title{
Orthodoxe Kirchenpresse in der Bundesrepublik Deutschland
}

\author{
von Edmund Plazinski
}

In den orthodoxen Kirchen des Ostens hat sich nur schwer und unter ungünstigen Voraussetzungen eine kirchliche Presse entwickelt. Ein theologischer Konservativismus $^{1}$, der nicht selten in den nach weltlichen Vorbildern ausgerichteten Massenkommumunikationsmitteln eine Gefährdung des charismatischen Lebens der Gemeinde sah², verhinderte den Aufbau eines Pressewesens, in dem sich die vielfältigen gesellschaftlichen und religiösen Strömungen innerhalb der Kirche widerspiegeln konnten. Mancherorts wurden derartige Versuche durch die politischen Umwälzungen eingeengt oder gar unterdrückt. Nach zwei Weltkriegen führte die ungewollte Ausbreitung der Orthodoxie auf westliche Länder zwangsläufig zu einer Überprïfung der bis dahin eingenommenen Standpunkte. Man wurde durch das Beispiel anderer Kirchen, in deren Nachbarschaft man rückte, angeregt, die Massenmedien intensiver in den Dienst der eigenen Sache zu stellen.

In der Bundesrepublik Deutschland stellt die orthodoxe Kirche eine leicht zu ibersehende Minderheit dar. Klammert man die Angehörigen der griechisch-orthodoxen Kirche aus, die als Gastarbeiter nur vorübergehend hier wohnen und die aller Voraussicht nach einmal in ihre Heimat zurïckkehren, so sind es etwa $60000^{3}$ Menschen, meist Emigranten aus ost- oder südosteuropäischen Ländern, die eine mehr oder weniger bodenständige orthodoxe Kirche bilden. ${ }^{4}$

Für viele orthodoxe Christen, die oft wochenlang, manchmal auch über Monate hinweg, keine Möglichkeit besitzen, an einem Gottesdienst ihrer Kirche teilzunehmen und die Predigt des Priesters zu hören, ist das gedruckte Wort die einzige Verbindung zur Kirche und zur Gemeinschaft in ihr.

Für die orthodoxe Kirche im Westen Deutschlands hätte sich im Laufe der Jahre das kirchliche Pressewesen eigentlich zu einem wesentlichen Faktor im religiösen Leben entwickeln müssen. Neben der Berichterstattung aus dem kirchlichen Leben, der Meinungsbildung und Meinungsäußerung, könnte durch sie die Unterweisung eine Bedeutung erlangen, die über die Zukunft der Kirche mitentscheidet. Die Diasporasituation der orthodoxen Kirchen führt nämlich auch dazu, daß die Alten weiter aus der Erinnerung leben, während die Jugend - durch Schule, Beruf, Umgebung, durch Freundschaften und Kontakte $\mathrm{zu}$ "westlichen " Glaubensgemeinschaften - immer mehr ihrer Kirche entgleitet und ihr schließlich verlorengeht. ${ }^{5}$

Dennoch erscheint es zweifelhaft, ob die orthodoxe Exilpresse in der Bundesrepublik überhaupt in der Lage ist, ein lebendiges Bindeglied zwischen dem einzelnen Christen und der Gemeinschaft und zum Träger der Verkündigung gegenüber jenen $\mathrm{zu}$ werden, die das Kanzelwort nicht erreicht. Es erweist sich, daß eben die gleichen Gründe, die einer geregelten Seelsorge entgegenstehen, auch die Versuche ergebnislos verlaufen lassen, mit einer "lautstarken" Kirchenpresse Entfernungen zu überwinden und die Kanzel näherzurücken.

Edmund Plazinski, Leiter der Presse- und Informationsabteilung der Steyler Mission in St. Augustin bei Bonn und freier Mitarbeiter, befaßt sich u. a. mit kirchenpolitischen und konfessionskundlichen Fragen unter besonderer Berücksichtigung der Ostkirchen. 
Die Isolation der orthodoxen Christen in der Bundesrepublik wird verstärkt durch ihre Zugehörigkeit zu verschiedenen Nationalkirchen. Der Mangel an Gemeinsamkeit im Kirchenraum überträgt sich spürbar auf die Kommunikationsmittel. Es zeigt sich eine Zersplitterung der Kräfte, die das Ziel gefährdet, das alle noch gemeinsam haben: die Bewahrung des orthodoxen Glaubens in einer fremden Welt.

Die meisten Ost-Emigranten in der Bundesrepublik müssen einer sozial schwachen Schicht zugerechnet werden. Die Renten- und Sozialempfänger bilden unter ihnen eine verhältnismäßig große Gruppe. Den Kirchen fehlen die gesetzlichen Möglichkeiten, Kirchensteuer zu erheben. ${ }^{\circ}$ Die freiwilligen Beiträge der Gläubigen decken nur zu einem geringen Teil die Aufwendungen für die Seelsorge, für den Unterhalt der Geistlichen und der kirchlichen Einrichtungen. Sie sind weitgehend auf die Unterstützung der Okumene angewiesen. Daß aus diesem bescheidenen Finanzaufkommen keine großen Aufwendungen zum Ausbau der Presse gemacht werden können, ist verständlich. Die Finanzierung der Kirchenblätter muß daher weitgehend durch das Abonnement gesichert werden. Die Zahl der Abonnenten ist gering. Sie bietet nur wenig Anreiz für die Wirtschaftswerbung. So bestimmt das Fehlen einer gesunden wirtschaftlichen Grundlage weitgehend Inhalt und Aufmachung, Druckqualität und Erscheinungsweise.

Eine Kirchenzeitung für alle sechs orthodoxen Jurisdiktionen in der Bundesrepublik, wie sie hin und wieder aus den eigenen Reihen angeregt wird, wäre nur möglich, wenn in dem Miteinander der Nationalkirchen ein Idealzustand erreicht würde.7

Die Kirchenblätter haben schließlich zur Zeit noch eine weitere Aufgabe zu erfüllen, die aus der Bedeutung der orthodoxen Kirche für die Bewahrung des Volkstums und der Muttersprache deutlich wird. Die einzelnen Landeskirchen sehen es als ihre Pflicht an, ihre Gläubigen auch außerhalb des Vaterlandes für die Nation zu erhalten. ${ }^{8}$ Die Kirche verkörpert in der Emigration auch ein Stück weltlicher Heimat und einen kulturellen Mittelpunkt. Damit verbinden sich dann auch politische und gesellschaftliche Vorstellungen, die nicht allein aus einer Gegnerschaft $z u$ jenem System erwachsen, das seit einem halben Jahrhundert oder seit mehr als zwei Jahrzehnten in der Heimat herrscht. Teile der russischen, rumänischen und der serbischen Kirche fühlen sich weiterhin der Monarchie und ihrem alten Herrscherhaus verbunden, wobei die Verbindung von Thron und Altar in eine fast dogmatische Höhe gerückt ist. All das findet nicht selten auch in den Zeitschriften seinen Niederschlag. Die Kirchenzeitung wird zu einem patriotischen Blatt und zur Verfechterin einer Staatsvorstellung, die durchaus nicht von allen Emigranten geteilt wird.

Man braucht nicht den Pessimismus führender orthodoxer Geistlicher zu teilen, die ihrer Kirche in Deutschland nur noch eine Lebensdauer von 10 bis 20 Jahren zugestehen und die Mitschuld daran den unzureichenden publizistischen Mitteln geben, um festzustellen, daß die Mehrheit der Kirchenzeitungen an ihren Lesern vorbeischreibt oder ihre Erwartungen nicht erfüllt. Die ältere Generation fühlt sich noch angesprochen. Die Jugend wird nicht mehr erreicht, weil sie gesellschaftlich bereits im Westen eingegliedert ist. Sie ist schnell bereit, in dem Anliegen jener kirchlichen Publizistik restaurative Tendenzen zu erkennen, die mit ihrer Auffassung von Kirche, Staat und Gesellschaft nicht übereinstimmen. Sie vermißt die Behandlung der Fragen, die sich dem modernen orthodoxen Menschen stellen, der sich in einer pluralistischen Gesellschaft $\mathrm{zu}$ behaupten hat und nach einer Führung durch die Kirche Ausschau hält.

In der Bundesrepublik werden vier orthodoxe kirchliche Zeitschriften mit einer Auflage von etwa 8000 Exemplaren (Stand vom 31. 12. 1968) herausgegeben. 
Zwei erscheinen in deutscher, eine in russischer und eine in ukrainischer Sprache. Die beiden letzteren sind Familienzeitschrift, Bistumszeitung, Nachrichtenbulletin, Landsmannschaftsorgan, Klerusblatt, kirchliches Amtsblatt und theologische Fachzeitschrift in einem. Keine wird von Journalisten oder journalistisch ausgebildeten Priestern redigiert. Man spürt die redliche Mühe der Gestalter; doch ist der Hang zum Erbaulichen oder zum Traktat unverkennbar. Der Vielschichtigkeit des Leserkreises wird selten Rechnung getragen.

Fünf weitere Zeitschriften, die außerhalb der Bundesrepublik erscheinen, sind als offizielle Organe ihrer Jurisdiktionen auch hier vertreten. Sie stammen aus Ost-Berlin (deutschsprachig), London, Paris und den USA. Zwei von ihnen (Ost-Berlin, Paris) werden von journalistisch ausgebildeten Priester-Redakteuren unter Mitarbeit von Berufsjournalisten gestaltet.

Drei Zeitschriften kommen monatlich heraus, eine alle zwei Monate, fünf vierteljährlich. Eine aktuelle Berichterstattung aus dem Leben der Kirche ist kaum gewährleistet. Das wird häufig den politischen Blättern der Emigranten-Organisationen überlassen. Wer regelmäßig und objektiv mit Nachrichten und Berichten aus der Gesamt-Orthodoxie versorgt werden will, greift zu Presse-Erzeugnissen anderer Kirchen. Selbst in der deutschen Tagespresse wird kontinuierlicher aus dem orthodoxen Kirchenleben berichtet.

Von den in der Bundesrepublik verlegten Zeitschriften verdienen zwei, hervorgehoben zu werden, weil die Herausgeber sich bemühen, das Anliegen ihrer Kirche auch den nichtorthodoxen Christen nahezubringen. Eine Voraussetzung dafür ist die deutsche Sprache. "Orthodoxie heute", herausgegeben in Düsseldorf von Erzpriester Sergius Heitz, einem ausgezeichneten Liturgiekenner, soll zum Verständnis der orthodoxen Theologie unter den Christen des Westens beitragen. Sie gilt nicht als "offizielle Stimme ${ }^{\text {und }}$ will auch nicht als Gemeindeblatt angesehen werden. Ihre Aussage berührt nur den theologischen Bereich. Das von Heitz begründete und ohne kirchenamtliche Förderung vertriebene Blatt wird außerhalb der Bundesrepublik vielfach als maßgebliche orthodoxe Meinung gewertet. ${ }^{{ }^{2}} Z$ wischen theologischer Zeitschrift und Kirchenzeitung stehen die in Wilhermsdorf erscheinenden "Ortho-

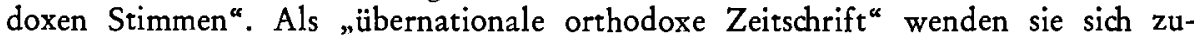
nächst an die deutschsprechenden Orthodoxen, dann auch an Andersgläubige, die die orthodoxe Kirche kennenlernen wollen. ${ }^{10}$

Im Mai 1961 erschien die erste Ausgabe der „Stimme der Orthodoxie", die vom mitteleuropäischen Exarchat des Moskauer Patriarchats in Berlin-Karlshorst (OstBerlin) für die Gläubigen in der DDR, der Bundesrepublik und in Ơsterreich herausgegeben wird.11 Beachtlich in Form und Inhalt, verzichtet auch sie darauf, Publikumszeitschrift $\mathrm{zu}$ sein. Im wesentlichen ist sie die deutschsprachige Ausgabe des "Journal des Moskauer Patriarchats". Es werden die amtlichen Beschlüsse und Verfügungen des Heiligen Synods der russisch-orthodoxen Kirche, Berichte aus dem Leben des Patriarchats, Predigten, theologische und kirchengeschichtliche Beiträge und Auseinandersetzungen veröffentlicht. Deutlich ist die Absicht zu spüren, mit den nichtorthodoxen Christen ins Gespräch zu kommen. Der Arbeit der Prager Christlichen Friedenskonferenz wird große Aufmerksamkeit geschenkt. Ergänzt wird der Inhalt der Zeitschrift jeweils durch eine Zusammenfassung der wichtigsten Ereignisse im Jurisdiktionsbereich des mitteleuropäischen Exarchats. Eine gründlichere und umfassendere Information aus der russisch-orthodoxen Kirche des Moskauer Patriarchats kann sowohl dem orthodoxen Christen deutscher Sprache wie auch dem Außen- 
stehenden kaum geboten werden. Die "Stimme der Orthodoxie" ist allerdings keine Zeitschrift, in der der Gläubige Antwort auf die Fragen findet, die sich ihm als Christ im Alltag stellen.

Die erheblichen qualitativen Unterschiede bei den orthodoxen Zeitschriften, die in der Bundesrepublik den Leser erreichen, ihre schwierige Aufgabe, die eingeengten Wirkungsmöglichkeiten und die begrenzte Reichweite sind selbstverständlich auch von orthodoxen Würdenträgern erkannt worden. Sie sehen ein, daß die orthodoxe Kirchenpresse in Deutschland den mannigfachen Schwierigkeiten nur entgehen kann, wenn sie sich zur Lösung ihrer Probleme zusammenfindet. ${ }^{12}$ Notwendig wäre vor allem eine deutlichere Abgrenzung und Bestimmung ihrer publizistischen Aufgaben. Besonders müßte eine Trennung von Kirchenzeitung (Bistumsblatt) und theologischer Fachzeitschrift herbeigeführt werden.

Archimandrit Johann Peterfalvy, einer der namhaften orthodoxen Theologen in Deutschland, verlangt von den Massenkommunikationsmitteln seiner Kirche außerdem, daran zu denken, daß die jungen Russen, Ukrainer, Serben, Rumänen und andere inzwischen deutsche Bürger geworden sind, die in ihrer Kirchenzeitung als deutsche orthodoxe Christen angesprochen werden wollen. Seiner Meinung nach muß sich eine orthodoxe Kirchenpresse in Deutschland bewußt in den Dienst der Okumene und der Weltkirche stellen. Daher komme ihr auch der Auftrag zu, Brückenbauer zwischen den Kirchen des Ostens und des Westens zu sein. Z.unächst aber sei es notwendig, so meint der Archimandrit, daß sie jene nationalen, pelitischen und jurisdiktionellen Eigenheiten aufgebe, die ihre Lebensfähigkeit bedrohen. In ihrer theologischen Haltung müsse sie sich aus dem Traditionellen lösen rind aufgeschlossen werden für die heutige Welt und deren Forderungen an die Kirche. ${ }^{13}$

\section{Anmerkungen:}

1. "Wohl mit unter dem Druck der politischen Entwicklung hat die Orthodoxie einen Überschwang zum Jenseitigen hin aufzuweisen, der sie an der spezifischen Aufgabe des Christen hindert, als ,Mitarbeiter Gottes', wie der Apostel Paulus sagt, die Welt im Geist Christi zu gestalten... So hat sich die Orthodoxie oft nur zu bereitwillig von der Aufgabe dispensiert, die Welt christlich zu gestalten, und hat sich gern damit getröstet, daß diese Welt sowieso bis zum Jüngsten Tag, im Argen liegt' “ Ernst Benz, Geist und Leben der Ostkirche, Hamburg 1957, S. 176.

2. Vgl. etwa den "Hirtenbrief des Bischofskonzils der Russisch-Orthodoxen Auslandskirche an die Gläubigen in der Fremde", New York, Oktober 1959, der die zerstörerische Macht der modernen technischen Erfindungen und den verhängnisvollen Einfluß der Massenkommunikationsmittel beklagt. In: Notes et Documents, Paris, Sonderausgabe des Centre Orthodoxe d'Informations, Paris, März 1960, 3.

3. Alex Proc in: Oekumenische Kommission für die Unterstützung orthodoxer Priester in der Bundesrepublik Deutschland (Hrsg.), Die Orthodoxen Exilkirchen in der Bundesrepublik Deutschland, München 1965, S. 5. - Paul Miron gibt die Zahl der orthodoxen Christen aus Osteuropa in der Bundesrepublik mit 50000 an: Paul Miron, Ostkirche im Exil, Teil I, gesendet im II. Programm des WDR Köln am 20. 3. 1966, 21.30 bis 22.10 Uhr; Manuskr. S. 7.

4. Vgl. auch: Edmund Plazinski, Sie leben mitten unter uns - Ein Bericht über die orthodoxen Exilkirchen in der Bundesrepublik, in: Katholisches Sonntagsblatt, Stuttgart, 115:1967, Nr. 36, S. 6, und Nr. 37, S. 6.

5. Erklärung von Archimandrit Johann Peterfalvy, München, vom 4. April 1967 (Vervielfältigtes Rundschreiben an kirchliche Stellen und Einzelpersonen in der Bundesrepublik)

6. Paul Miron, Ostkirche im Exil, Sendemanuskript Teil II, S. 11; gesendet am 24. 3.1966 von 21.50 bis 22.30 Uhr. Vgl. Anm. 3.

7. Brief von Archimandrit Peterfalvy, München, an den Verfasser, vom 7. März 1968.

8. Vgl. Metropolit Ilarion, Chvalimo Boha ukrajinski movoju ( ukrainischen Sprache"), Winnipeg 1962, S. 74; und Ernst Benz, a.a.O. 
9. „Orthodoxie heute": Daten im Anhang.

10. Die erste Nummer erschien im Januar 1954 in Form einiger vervielfältigter Blätter, herausgegeben und unter der Redaktion von Vera Kreger mit Gutheißung des russischorthodoxen (Emigranten-) Bischofs Averky (jetzt Jordanville/New York). Daten im Anhang.

11. Im Vorwort der ersten Ausgabe schreibt Exarch Ioann Wendland: „Als Presseorgan des Exarchats will die Zeitschrift ,Stimme der Orthodoxie' ihre Leser mit einzelnen Zügen aus dem Leben der Russischen Orthodoxen Kirche, vor allem aber mit dem innerkirchlichen Geschehen der orthodoxen Gemeinden in Deutschland sowie mit dem Leben und der Tätigkeit des Mitteleuropäischen Exarchates bekannt machen. Gleichzeitig sieht die Zeitschrift ihre Aufgabe darin, zur Festigung von Kontakten zwischen der Russischen Orthodoxen Kirche und allen christlichen Kirchen, religiösen Organisationen und Gemeinschaften innerhalb des Mitteleuropäischen Exarchates beizutragen. ${ }^{\star}$

12. "Wir können es uns einfach nicht leisten, kirchliche Aufgaben mit politischen Tendenzen zu vermengen, zumal wir in allen Glaubensthesen völlig einig sind. Und wir müssen an die Zukunft unserer Gemeinden denken." Johann Peterfalvy, Gegenwart und Zukunft der Orthodoxie in Westeuropa, in: "Orthodoxe Stimmen" 14:1967, Nr. 56, S. 4.

13. Brief von Archimandrit Peterfalvy, München, an den Verfasser, vom 7. 3. 1968.

\section{Anhang:}

\section{In der Bundesrepublik Deutschland verlegte orthodoxe Zeitschriften}

"Cerkovnyja Wedomosty" [Kirchliche Bekanntmachungen] Sprache: Russisch. Herausgeber: Diözesanverwaltung der russisch-orthodoxen Auslandskirche in Deutschland. Verlagsort: München (8 München 9, Zeppelinstraße 3). Erscheinungsweise: vierteljährlich. Herstellung: Buchdruck. Format DIN A 4, 40 Seiten pro Heft. 1. Jahrgang 1951.

Die Zeitschrift gilt als Bistumsblatt für die Bundesrepublik. Sie wird von Priestern redigiert. In ihrem Inhalt beschränkt sie sich hauptsächlich auf Nachrichten aus dem Bereich der Auslandskirche.

"Ridna Cerkwa" [Die Heimatkirche] Sprache: Ukrainisch. Herausgeber: Ukrainisch-orthodoxes theologisches wissenschaftliches Institut. Verlagsort: Karlsruhe (75 Karlsruhe-Durlach, Postfach 9). Druckort: Neu-Ulm. Herstellung: Buchdruck. Format: 21,4 mal $29,5 \mathrm{~cm}$. 20 Seiten pro Heft. 1. Jahrgang 1952. Erscheinungsweise vierteljährlich. Auflage: ca. 2000.

„Ridna Cerkwa ${ }^{\alpha}$ ist das Bistumsblatt der ukrainischen Kirche für Deutschland (Auslandskirche). Sie wird redigiert von einem Kollegium aus Priestern, Professoren und Laien. In ihrem Aufbau entspricht sie den katholischen Bistumsblättern. Auffallend sind die zahlreichen theologischen Beiträge, die sich an den Priester und den gebildeten Laien wenden. Stark national betont.

„Ortbodoxie bente" Sprache: Deutsch. Herausgeber: Erzpriester Sergius Heitz. Verlagsort: Düsseldorf (4 Düsseldorf, Pattscheider Straße 30). Erscheinungsweise: vierteljährlich. Herstellung Rotaprint, farbiger Umschlag im Buchdruck bei gleichem Motiv. Format: DIN A 4. 22-26 Seiten pro Heft. 1. Jahrgang 1962. Auflage: ca. 3000.

Erzpriester Heitz untersteht der Jurisdiktion der rumänischen Auslandskirche innerhalb der russisch-orthodoxen Auslandskirche. Seine Zeitschrift ist kein offizielles Organ. Sie beschränkt sich auf theologische Fragen mit Blickrichtung auf den Christen des Westens. Kein ausgeprägter Nachrichtenteil. Nicht für eine breitere Publikumsschicht bestimmt.

„Orthodoxe Stimmen“ Sprache: Deutsch. Herausgeber: Archimandrit Johann Peterfalvy, München. Verlagsort: Wilhermsdorf (8534 Wilhermsdorf, Ansbacher Straße 18). Druckort: Stuttgart. Erscheinungsweise: vierteljährlich. Herstellung: Rotaprint. Format DIN A 5. 26 Seiten pro Heft. 1. Jahrgang 1954. Auflage: 1000. - Neueste Daten: S. 222.

Die lange Zeit von der Laientheologin Vera Kreger unter Mitarbeit orthodoxer Theologen redigierte Zeitschrift wird als übernational bezeichnet. Viele Beiträge sind Übersetzungen aus ausländischen orthodoxen Zeitschriften aller Nationalitäten. Der Nachrichtenteil ist bescheiden. In der theologischen Aussage bleibt sie für den Laien verständlich, dem sie Ersatz für ein deutschsprachiges Gemeindeblatt sein will. Zahlreiche religiöse Beiträge mit erbaulichem Charakter. Archimandrit Peterfalvy, seit Herbst 1967 Herausgeber, ist Bevollmächtigter der 
orthodoxen Kirche von Frankreich und Westeuropa in der Bundesrepublik, einer autonomen Kirche, die bis zum Frühjahr 1967 als russisches Exarchat dem ökumenischen Patriarchen unterstand. Neuer Stand 1. 7. 1969: Im Frühjahr 1969 ist die Laientheologin Vera Kreger aus der Redaktion der Zeitschrift ausgeschieden. Der Verlagsort wurde von Wilhermsdorf nach München 71, Engadiner Straße 20, verlegt. Die ersten beiden Nummern des 16. Jahrgangs zeigen eine deutliche Anderung in der redaktionellen Linie. Die Beiträge sind sachlicher geworden, der Nachrichtenteil aus der orthodoxen Welt nimmt einen größeren Raum ein. Ende März 1969 erschien die erste Nummer (1. Vierteliahr 1969) einer neuen, von Vera Kreger herausgegebenen Vierteljahreszeitschrift mit dem Titel "Orthodoxe Rundschau" und dem Untertitel "Auszüge aus orthodoxem religiösem Schrifttum aus verschiedenen Ländern und Jurisdiktionen". In dieser Zeitschrift, die in Format und Aufmachung den "Orthodoxen Stimmen" gleicht, setzt Vera Kreger ihre bisherige Arbeit fort. In der ersten Nummer schreibt sie: "Die Zeitschrift ,Orthodoxe Stimmen', die von der Verfasserin vor 15 Jahren gegründet und 14 Jahre lang geleitet wurde, hat bereits vor längerer Zeit versuchit, sich von der Bindung an eine Jurisdiktion zu lösen. Sie war aber durch die für die Zeitschrift verantwortlichen Geistlichen mindestens dem äußeren Anschein nach doch an eine bestimmte Jurisdiktion gebunden. Das machte ihre Neutralitätsbestrebungen in den Augen vieler orthodoxer Leser wenn nicht zunichte, so doch zweifelhaft. Viele haben deshalb die Zeitschrift abbestellt, manchmal sogar mit deutlichen Außerungen ihres Mißfallens. Wir haben eingesehen, daß es zwei Arten von Neutralität gibt. Die eine möchten wir Neutralität der Verneinung nennen (wir gehören zu keiner der orthodoxen Jurisdiktionen) - sic war offenbar auf die Dauer nicht zu verwirklichen. Die zweite dagegen ist eine Neutralität der Bejahung (wir gehören zu allen orthodoxen Jurisdiktionen); diese Art der Neutralität soll die neue Zeitschrift ,Orthodoxe Rundschau' vertreten." (Orthodoxe Rundschau, 1:1969, Nr. 1, Seite 3-4).

\section{Außerhalb der Bundesrepublik verlegte, aber hier verbreitete orthodoxe Zeitschriften}

„Stimme der Orthodoxic ${ }^{\alpha}$ Sprache: Deutsch. Herausgeber: Mitteleuropäisches Exarchat des Moskauer Patriarchats. Verlagsort: Ost-Berlin. (Berlin-Karlshorst, Wildensteiner Straße 10). Erscheinungsweise: monatlich. Herstellung: Buchdruck. Format: 16,8 mal 24,2 cm. 66 Seiten pro Heft. 1. Jahrgang 1961.

"Stimme der Orthodoxie" ist die offizielle Kirchenzeitung des mitteleuropäischen Exarchats des Moskauer Patariarchats, das Deutschland und Osterreich umfaßt. Die Zeitschrift wird von einem journalistisch vorgebildeten Priester-Redakteur unter Mitarbeit von Fachleuten gestaltet. Die meisten Beiträge stammen aus dem "Journal des Moskauer Patriarchats“.

„Glas ${ }^{\star}$ [Die Stimme] Sprache: Serbisch. Herausgeber: Bruderschaft der serbisch-orthodoxen Kirche für Westeuropa. Verlagsort: London. (12, Egerton Gardens, London S.W. 3.). Erscheinungsweise: alle zwei Monate.

„Glas" gilt als offizielle Kirchenzeitung für ganz Westeuropa. National eingestellt mit monarchistischen Tendenzen.

„Cerkovnyi Vestnik ${ }^{\alpha}$ [Kirchlicher Bote] Sprache: Russisch. Herausgeber: Orthodoxe Kirche von Frankreich und Westeuropa. Verlagsort: Paris (12, Rue Darue, Paris 8). Erscheinungsweise: monatlich.

Diese Zeitschrift versucht, in ihrem Inhalt der Tatsache Rechnung zu tragen, daß sich die Gläubigen im Exil in ihrer Lebenshaltung weitgehend der neuen Umgebung angepaßt haben. Es sind Pläne vorhanden, sie in französischer Sprache erscheinen zu lassen. Die Zeitschrift gilt zur Zeit auch als Organ für die Kosaken in der Bundesrepublik. Sie erscheint unter Mitarbeit von Fachjournalisten. Als Publikumszeitschrift soll sie einen breiten Leserkreis erreichen.

„Cerkowny Prawoslawy Biuleten ${ }^{*}$ [Orthodoxes Kirchenblatt] Sprache: Russisch. Herausgeber: Bischof Matthäus, Ordinarius der polnisch-orthodoxen Christen im Jurisdiktionsbereich des griechisch-orthodoxen Erzbistums von Thyateira und Großbritannien (Westeuropa). Verlagsort: London (7 Penywern Rd., London S. W. 5.). Erscheinungsweise: vierteljährlich.

Das „Orthodoxe Kirchenblatt “ kam bis 1954 zweimal monatlich heraus. Aus finanziellen Gründen wurde die Erscheinungsweise geändert. In seinem Inhalt soll es Gemeindeblatt, Amtsblatt und theologische Zeitschrift sein. Um die polnischen Orthodoxen verschiedener Muttersprachen (polnisch, ukrainisch, weißrussisch) zu erreichen, wurde die russische Sprache gewählt. 
"Jumala Abiga [Mit Gottes Hilfe] Sprache: Estnisch. Herausgeber: Erzpriester Sergius Samon. Verlagsort: Los Angeles (5332 Fountain Avenue, Los Angeles, Calif. USA). Erscheinungsweise: monatlich.

Die Kirchenzeitung der estnischen orthodoxen Kirche im Exil unter der Jurisdiktion des ökumenischen Patriarchen erscheint in den USA, weil sie hier in den zahlenmäßig größeren Gemeinden mit wohlhabenderen Gläubigen eine bessere wirtschaftliche Grundlage findet. Neben ihren Aufgaben als Bistumsblatt und theologische Zeitschrift unterstützt sie vor allem die nationalen Belange der Esten, wie die Forderung nach Unabhängigkeit von der Sowjetunion. Sie wird nicht von Fachjournalisten gestaltet, bringt aber gelegentlich Beiträge von Journalisten.

\section{S U M M A R Y}

In the Federal Republic of Germany there are about 60000 Orthodox Christians from east and southeast European countries. They belong to six different ecclesiastical jurisdictions. For these Christians there are four quarterlies, two in German, one in Russian and one in Ukrainian. The two latter are official publications of their respective churches. Outside the Federal Republic there are five other church papers (one in German, two in Russian, one in Serbian and one in Esthonian) devoted to the faithful in West Germany. Three of these are published monthly, one every second month and one quarterly. All these papers reach only a small number of the people they should reach, because they fail to appeal especially to the young people, who are mud more at home in the new society in which they are living than are their parents, who came from the various Orthodox-rite countries. It must be added that only about two of the papers mentioned fulfill the requirements of a good journalistic standard. The idea of putting out just one well done paper in common seems not to be welcome to the people responsible.

\section{RESUMEN}

En la República Federal Alemana viven cerca de 60000 cristianos ortodoxos procedentes de países del este o sudeste europeo. Pertenecen a 6 jurisdicciones eclesiásticas diferentes. Trimestralmente aparecen cuatro revistas: dos en alemán, una en ruso y otra en ucraino; las dos últimas son brganos oficiales de sus respectivas Iglesias. Fuera de la República Federal se publican otros cinco periódicos eclesiásticos (uno en alemán, dos en ruso, uno en serbio y otro en estoniano) destinados a los fieles de Alemania Occidental. Tres de ellos aparecen mensualmente, dos bimensualmente y otro cada cuatro meses. El número de sus lectores es muy reducido, ya que no toman en cuenta el hecho de que, tanto los emigrados como sus hijos, se han adaptado a su nuevo ambiente, disminuyendo con ello las tendencias nacionalistas. Cabe añadir, además, que, desde el punto de vista periodístico, solamente dos de dichas publicaciones están a la altura de su cometido. El deseo de los ortodoxos que viven en Alemania es el de poseer un periódico de salida más frecuente y que, éste, no solamente les tenga al corriente de 10 que sucede en el mundo ortodoxo, sino que tenga también en cuenta la asimilación que los ortodoxos hacen del ambiente y mentalidad de la nación que les ha ofrecido hospitalidad. Esta sugerencia, sin embargo, halla poca o ninguna comprensión por parte de las autoridades eclesiásticas. No quieren renunciar a su idiosincrasia nacional, política y jurisdiccional, no percatándose del peligro a que exponen con ello a la capacidad vital de toda la orthodoxía. 